\title{
Low-Income Parents' Perceptions of and Engagement With a Digital Behavioral Training Program: Mixed Methods Study
}

\author{
Jenna Brager ${ }^{1 *}$, MS, PhD, BSN-RN; Susan Breitenstein ${ }^{2 *}$, BSN, RN, FAAN, PhD; Deborah Gross ${ }^{3 *}$, BSN, RN, FAAN, \\ DNSC; Hailey Miller ${ }^{3 *}$, BSN-RN
}

\footnotetext{
${ }^{1}$ LifeBridge Health - Sinai Hospital of Baltimore, Baltimore, MD, United States

${ }^{2}$ Ohio State University, School of Nursing, Columbus, OH, United States

${ }^{3}$ Johns Hopkins University, School of Nursing, Baltimore, MD, United States

${ }^{*}$ all authors contributed equally
}

\section{Corresponding Author:}

Jenna Brager, MS, PhD, BSN-RN

LifeBridge Health - Sinai Hospital of Baltimore

$2401 \mathrm{~W}$ Belvedere Ave

Baltimore, MD

United States

Phone: 4106018742

Email: jbrager@lifebridgehealth.org

\section{Abstract}

Background: Parent training is a method for strengthening parenting skill, reducing child behavior problems, and promoting positive parent-child relationships. However, few parents have access to these evidence-based programs. The ezParent program, a tablet-based delivery adaptation of the group-based Chicago Parent Program (CPP), is a parent training program designed to address the needs of families raising young children in urban poverty. There is extensive evidence that positive parenting practices can be a powerful buffer against the negative effects of poverty and adversity and is one of the strongest predictors of children's social and behavioral well-being.

Objective: This study aimed to explore (1) parents' perceptions of the benefits and barriers associated with their use of the ezParent program; and (2) the ways in which the ezParent components and perceived usability varied by program use (module completion).

Methods: This study uses an explanatory mixed-method design. Data were collected from 92 participants recruited from two pediatric primary care clinics (PPC) based in two urban cities with a high proportion of low income and minority families: Chicago, Illinois (cohort 1) and Baltimore, Maryland (cohort 2). Cohort $1(n=42)$ was recruited between October 2013 and June 2014 as part of a randomized controlled tirla. Cohort $2(n=50)$ was recruited between May 2017 and July 2017 as part of a single group design. The current report focuses on a subsample from cohorts 1 and 2 who were interviewed about their experiences using the program. To explore parents' perceptions of ezParent (ie, perceived usefulness of content, ease of use, barriers to use, qualities affecting interest, and intention), all participants were invited to participate in an individual interview after the $12^{\text {th }}$ week. Based on degree of module completion, we grouped parents into two user groups: high (completed 4-6 modules) and low (completed 0-3 modules). This cut-off was chosen based on previous data showing that parents who attended at least $50 \%$ of the CPP group sessions reported greater improvements in parenting self-efficacy, use of discipline, and warmth towards their children, as well as fewer child behavior problems.

Results: Fifty-nine parents participated in follow-up interview: 32 (54.24\%) from cohort 1 and 27 (45.76\%) from cohort 2. Among those interviewed, 23 (38.9.5\%) parents completed all six modules and 12 parents (20.3\%) completed none of the modules. Specifically, among those interviewed, parents completed an average of 3.73 (SD 2.39) modules compared to an average of 1.79 (SD 2.38) modules completed among those not interviewed. Parents who completed more modules reported more program benefits and those who completed fewer modules reported more barriers.

Conclusions: Exploring users' experience with current digital applications, researchers and application developers can better design future tablet-based interventions to be both effective and acceptable by end users. Furthering our understanding of factors associated with engagement will inform more effective tailoring and improvements of mHealth interventions, particularly those targeting vulnerable populations. 
(iproc 2019;5(1):e15090) doi: $\underline{10.2196 / 15090}$

\section{KEYWORDS}

mobile health (mHealth); mobile health intervention; parent-child relations; digital health

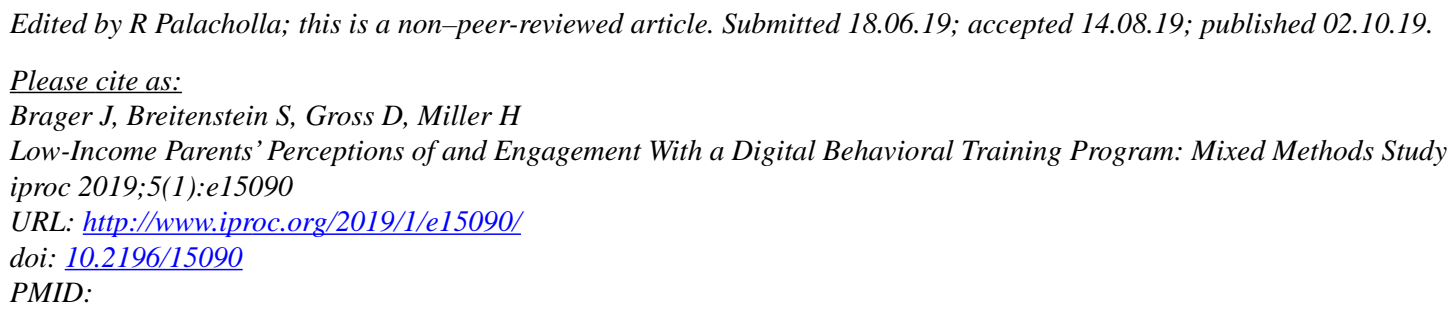

CJenna Brager, Susan Breitenstein, Deborah Gross, Hailey Miller. Originally published in Iproceedings (http://www.iproc.org), 02.10.2019 This is an open-access article distributed under the terms of the Creative Commons Attribution License (https://creativecommons.org/licenses/by/4.0/), which permits unrestricted use, distribution, and reproduction in any medium, provided the original work, first published in Iproceedings, is properly cited. The complete bibliographic information, a link to the original publication on http://www.iproc.org/, as well as this copyright and license information must be included. 